\title{
An apoptosis targeted stimulus with nanosecond pulsed electric fields (nsPEFs) in E4 squamous cell carcinoma
}

\author{
Wei Ren $\cdot$ Stephen J. Beebe
}

Published online: 7 January 2011

(C) The Author(s) 2011. This article is published with open access at Springerlink.com

\begin{abstract}
Stimuli directed towards activation of apoptosis mechanisms are an attractive approach to eliminate evasion of apoptosis, a ubiquitous cancer hallmark. In these in vitro studies, kinetics and electric field thresholds for several apoptosis characteristics are defined in E4 squamous carcinoma cells (SCC) exposed to ten $300 \mathrm{~ns}$ pulses with increasing electric fields. Cell death was $>95 \%$ at the highest electric field and coincident with phosphatidylserine externalization, caspase and calpain activation in the presence and absence of cytochrome $c$ release, decreases in Bid and mitochondria membrane potential $(\Delta \psi \mathrm{m})$ without apparent changes reactive oxygen species levels or in Bcl2 and Bclxl levels. Bid cleavage was caspase-dependent (55-60\%) and calcium-dependent (40-45\%). Intracellular calcium as an intrinsic mechanism and extracellular calcium as an extrinsic mechanism were responsible for about 30 and $70 \%$ of calcium dependence for Bid cleavage, respectively. The results reveal electric field-mediated cell death induction and progression, activating pro-apoptoticlike mechanisms and affecting plasma membrane and intracellular functions, primarily through extrinsic-like pathways with smaller contributions from intrinsic-like pathways. Nanosecond second pulsed electric fields trigger heterogeneous cell death mechanisms in E4 SCC populations to delete them, with caspase-associated cell death as a predominant, but not an unaccompanied event.
\end{abstract}

W. Ren · S. J. Beebe $(\square)$

Frank Reidy Research Center for Bioelectrics, Old Dominion

University, 4211 Monarch Way suite 300, Norfolk,

VA 23508, USA

e-mail: sbeebe@odu.edu

W. Ren

Department of Biological Sciences, Old Dominion University, Norfolk, VA 23508, USA
Keywords Cell death - Cytochrome $c$. Mitochondria membrane potential $\cdot$ Caspase $\cdot$ Calpain $\cdot$ Calcium . Bcl2 - Bclxl · Phosphatidylserine externalization · Propidium iodide $\cdot$ Apoptosis pathways

\section{Introduction}

The administration of nanosecond second pulsed electric fields (nsPEFs) is emerging as a novel stimulus for inducing cell death. Apoptosis can be activated by nsPEFs in various cancer cell lines in vitro [1-3], a fibrosarcoma tumor ex vivo [1, 4-6] and in B16f10 melanoma tumors in vivo [7]. Apoptosis is a highly conserved mechanism in eukaryotes for eliminating superfluous, aberrant and damaged cells. It is a complex cascade of events morphologically characterized by phosphatidylserine externalization, cell shrinkage, chromatin condensation, nucleosomal DNA fragmentation, disruption of mitochondrial membrane potential $(\Delta \psi \mathrm{m})$ resulting in plasma membrane blebbing and formation of apoptotic bodies [8]. These morphological features are believed to be caused at least in part by activation of caspases, a family of cysteine proteases, which plays a crucial role to initiate and execute apoptosis [9-11], although other proteases may also be activated.

Caspases can be triggered through extrinsic death receptor pathways or intrinsic pathways depending on the cell type and stimulus $[12,13]$. Extrinsic pathway are initiated by specific ligands, such as Fas and TRAIL, which aggregate their cognate death receptor, allowing assembly of death-inducing signaling complexes (DISC) on the cytoplasmic side of plasma membranes leading to initiator caspase-8 (and/or -10) activation. In type I cells, caspase-8 activates executioner caspases independent of mitochondria. In type II cell pathways, caspase-8 cleaves the BH3-only 
protein Bid to activate pro-apoptotic truncated Bid (t-Bid). Translocation of t-Bid triggers cytochrome $c$ release from mitochondria and caspase- 9 activation through formation of the apoptosome, which causes cell demise through activation of caspase- 3 .

Unlike conventional electroporation, which primarily affects plasma membranes [14, 15], nsPEFs also electroporate intracellular membranes [16]. Modeling studies [14, $15,17,18]$ and evidence determined experimentally by patch clamp and fluorescent imaging studies [19, 20], indicated that nsPEFs with sufficiently short pulse durations and rapid rise times [16] induced nanopores in plasma membranes that were often smaller than propidium iodide (PI). This supra-electroporation involved all cell membranes going through cells, extensively penetrating all organellar membranes $[14,15]$. Effects on intracellular vesicles were observed without measurable effects on plasma membranes $[16,21]$ with PI or calcein and on intracellular calcium mobilization from the endoplasmic reticulum and capacitative calcium entry in HL-60 cells [22], suggesting that these external electric fields can mimic ligand signaling [5]. It is now know that the absence of effects on plasma membranes was due to the presence of nano pores that were too small to allow entry of the membrane markers or that detection systems were insufficiently sensitive to measure them $[19,20]$. Nevertheless, modeling evidence suggests that under certain circumstances it is possible to have effects on intracellular membranes without effects on the plasma membrane [18].

Studies have suggested that nsPEF-induced apoptosis mechanisms are cell type-dependent. In Jurkat cells caspase activation and cytochrome $c$ release were nearly coincident, making it difficult to differentiate between extrinsic and intrinsic mechanisms [1]. In B16f10 cells cytochrome $c$ was not released, implicating the extrinsic apoptosis pathway typical of type I cells [2]. In HCT 116 cells, caspase activation occurred before cytochrome $c$ release, suggesting activation of extrinsic pathways followed by mitochondria-mediated cytochrome $c$ release through the intrinsic pathway, type II cell extrinsic apoptosis pathway and/or feed-forward amplification from the initial extrinsic signal [3]. However, specific contributions from extrinsic or intrinsic mechanisms have not been determined.

Available data indicate pervasive effects of nsPEF on all cell membranes inducing apoptosis signaling in several cell types. However, their mechanisms, sites of action and unambiguous confirmation of apoptosis pathways contributing to cell death have not been clearly determined. In this study, we demonstrate direct activation of apoptosis signaling through multiple mechanisms and quantify relative contributions from several apoptosis pathways originating from both plasma membranes and intracellular membranes that exhibit both dependence and independence of caspases, calpains, calcium, and cytochrome $c$ release. Several quantified nsPEF-induced biological functions exhibit different electric field sensitivities and kinetics that promote heterogeneous apoptosis characteristics in E4 squamous carcinoma cells (SCC). Diverse effects of nsPEFs on different E4 SCC death mechanisms and programmed pathways can induce cell death by multiple mechanisms bypassing apoptosis-deficient syndromes in SCC.

\section{Experimental procedures}

Cells and cell culture

The E4 squamous cells were obtained from Dr. Jian-Hua Mao (Lawrence Berkeley National Laboratories) and were maintained in Dulbecco's modified Eagle's medium (ATCC) supplemented with $10 \%$ fetal bovine serum (FBS, Atlanta Biologicals), $2 \mathrm{mM}$ L-glutamine (Mediatech Cellgro), and $1 \%$ Penicillin-Streptomycin solution (Mediatech Cellgro). The cells were cultured in a humid atmosphere containing 5\% $\mathrm{CO}_{2}$ and $95 \%$ air at $37^{\circ} \mathrm{C}$ until reaching $50-75 \%$ confluency with passage ranging between 11 and 19 .

Induction of cell death by nanosecond pulsed electric fields

Cells were harvested with trypsin and re-suspended in fresh DMEM medium with $10 \%$ FBS to a concentration of $7.7 \times$ $10^{6}$ cells $/ \mathrm{ml} .130 \mu \mathrm{l}$ of cell suspension $\left(1 \times 10^{6}\right.$ cells $)$ were placed in $0.1 \mathrm{~cm}$ gap cuvettes (Biosmith) and exposed to ten pulses with $300 \mathrm{~ns}$ duration and electric field strength from 0 to $60 \mathrm{kV} / \mathrm{cm}$. The pulse power devise was previously described [3]. The energy absorbed or the dose $(\mathrm{J} / \mathrm{g})$ was calculated as previously described [23]. For all cell responses, time courses were conducted to determine time for maximal responses. Cell responses at all electric fields were stable for at least $90 \mathrm{~min}$.

\section{Determination of $24 \mathrm{~h}$ cell survival}

After treatment, E4 cells were seeded in 6-well plates $\left(3.3 \times 10^{5}\right.$ cells/well). Twenty-four hours later, cells were detached and trypan blue negative cells were counted. The cell numbers were normalized to control cells as $100 \%$.

Assessment of caspases-3 \& -7, -8, -9 activation by flow cytometry

Caspase- 3 \& -7,-8, -9 activation were assayed using a commercial method based on fluorochrome-labeled, irreversible caspase inhibitors (FLICA, Immunochemistry 
Technologies LLC). E4 cells were treated with different nsPEF conditions as mentioned above. One hour after treatment, cells were gently removed from cuvettes and resuspended in DMEM without FBS. According to the manufacturer's recommendations, cells were labeled with carboxyfluorescein caspase-3 \& $-7,-8,-9$ irreversible inhibitors for $1 \mathrm{~h}$ at $37^{\circ} \mathrm{C}$ under $5 \% \mathrm{CO}_{2}$ and protected from light as previously described [2]. Cells were washed with buffer to remove the unbound reagent. Cell fluorescence was determined by Becton-Dickinson FacsAria. Ten thousand cells were acquired for analyses and expressed as percentage of cells showing positive fluorescence. As electric fields were increased, greater numbers of cells became caspase positive with homogeneous shifts of cells into the caspase positive gate, making cell percentages with active caspases the most accurate and meaningful quantification of active caspases as an apoptosis marker.

Evaluation of active pan caspase by flow cytometry

E4 cells were exposed to specific nsPEF conditions as previous described and incubated with $40 \mu \mathrm{M}$ fluorescent-labeled, cell permeable, irreversible inhibitor FITC-VAD-fmk $\left(\mathrm{CaspACE}^{\mathrm{TM}}\right.$, Promega) for $20 \mathrm{~min}$. Cells were washed once with $1 \mathrm{ml}$ PBS and resuspended in $500 \mu \mathrm{l}$ PBS $1 \mathrm{~h}$ post pulse for flow cytometric analysis with Becton-Dickinson FACS Aria.

Measurement of cytochrome $c$ release by two different assays

Cytochrome $c$ release was measured by two different methods, which gave similar results (data not shown). In method I cytochrome $c$, which was used in all studies here, was assessed using the Innocyte Flow Cytometric Cytochrome $c$ Release assay (Calbiochem). One hour after nsPEF treatment, cells were permeabilized, fixed and washed. Cells were then incubated with primary antibody overnight at $4{ }^{\circ} \mathrm{C}$ and secondary antibody for $1 \mathrm{~h}$ at room temperature. Cells $(10,000)$ were then analyzed by BectonDickinson FacsAria flow cytometer. Method II data is not shown but was carried out as previously described [2,3]. Briefly, after treatment cells were incubated with a monoclonal anti-cytochrome $c$ antibody for $30 \mathrm{~min}$, washed with PBS, incubated with Alexa-Fluor-488 goat anti-mouse (Molecular Probes) for $30 \mathrm{~min}$, washed again and analyzed flow cytometry.

Measurement of mitochondrial membrane potential $(\Delta \psi \mathrm{m})$

Depolarization of $\Delta \psi \mathrm{m}$ was detected using a fluorescent lipophilic cation, tetramethylrhodamine ethyl ester
(TMRE) (Immunochemistry Technologies LLC). Cells were incubated with $200 \mathrm{nM}$ TMRE for $10-20 \mathrm{~min}$ at $37^{\circ} \mathrm{C}$ 30 min post pulse. Twenty minute incubations were suggested by the manufacturer, but $10 \mathrm{~min}$ incubations, which gave similar results, were used in some experiments to determine the earliest response. At the end of incubation, cells were re-suspended in assay buffer. The average intensity of red fluorescence (10,000 cells) was analyzed on the FL-2 channel of a Becton-Dickinson FacsAria flow cytometer. When JC-1 was used, cells were pulsed and incubated with the JC-1, washed and analyzed by flow cytometry as previously described [2]. For JC-1, red fluorescent aggregates change to green fluorescent monomers when $\Delta \psi \mathrm{m}$ decreases.

\section{Determination of phosphatidylserine externalization}

External phosphatidylserine exposure and plasma membrane permeability was evaluated by double-labeling cells with FITC-Annexin-V and PI using a detection kit (BD Pharmingen Biosciences). After administration of nsPEFs, cells were re-suspended in binding buffer and incubated with FITC-Annexin-V and PI according to the manufacture's procedures. The fluorescence signal was quantified by flow cytometry using FL1 (Annexin-V) and FL2 (PI) channels 15 min post pulse.

Assessment of cytochrome $c$ release with administration of $z$-VAD-fmk

Cells were preloaded with $100 \mu \mathrm{M}$ of $\mathrm{z}$-VAD-fmk for $30 \mathrm{~min}$ and followed by exposing cells to ten $300 \mathrm{~ns}$ pulses with 0 and $60 \mathrm{kV} / \mathrm{cm}$ electric fields. Cytochrome $c$ monoclonal antibody (BD Pharmingen Biosciences) and Alexa-Fluor 488 goat anti-mouse antibody (Invitrogen) were added to cell suspensions $1 \mathrm{~h}$ after pulsing as described previously [2]. Flow cytometric analysis of fluorescent intensity was done by using Becton-Dickinson FacsAria.

\section{Immunoblot analysis for Bcl-2, Bcl-xl, Bid and t-Bid}

For each antigen samples were taken for immunoblot analysis 0 15, 30 and 60 min after pulsing. Bcl2 and Bcl-xl data are not shown. At the indicated times E4 cells were washed twice with cold PBS and lysed in RIPA buffer (25 mM Tris- $\mathrm{HCl} \mathrm{pH}$ 7.6, $150 \mathrm{mM} \mathrm{NaCl}, 1 \% \mathrm{NP}-40,1 \%$ sodium deoxycholate, $0.1 \%$ SDS) (Pierce) for $30 \mathrm{~min}$ on ice. Then cell lysates were centrifuged at $15,000 \times g$ for $20 \mathrm{~min}$ at $4^{\circ} \mathrm{C}$ and protein content in the supernatant was measured using the BCA method (Pierce). Lysates were then boiled with $4 \times$ DualColor $^{\mathrm{TM}}$ Protein Loading Buffer 
(Fermentas Life Sciences) for $5 \mathrm{~min}$. Protein extracts $(75 \mu \mathrm{g})$ were separated by electrophoresis on $15 \%$ SDS-polyacrylamide gel electrophoresis (SDS-PAGE) and proteins were transferred onto a PVDF membrane with pore size of $0.2 \mu \mathrm{m}$ (Pierce, Rockford, IL). Membranes were blocked in Odyssey blocking buffer (LI-COR Biosciences) for $1 \mathrm{~h}$, and incubated overnight at $4{ }^{\circ} \mathrm{C}$ with the respective primary antibody directed against Bcl-2 (1:200 mouse polyclonal, Santa Cruz Biotechnology), Bcl-xl (1:1000 rabbit monoclonal, Cell Signaling Technology), or Bid/t-Bid (1:100 mouse monoclonal, R\&D System) diluted in blocking buffer. Membranes were washed three times with $0.1 \%$ Tween-20 in PBS and then incubated with an IRDye680-conjugated secondary antibody (LI-COR Biosciences) in blocking buffer for $1 \mathrm{~h}$. Protein-antibody complexes conjugated with IRDye 680 were quantified on the Odyssey Infrared Imaging System (LI-COR Biosciences). $\beta$-Actin (1:1000; Li-Cor Biosciences) was used as loading control.

Assessment of inhibitory effects of z-VAD-fmk, EGTA and BAPTA on nsPEFs induced-Bid cleavage

E4 cells were preincubated with $100 \mu \mathrm{M} \mathrm{z}$-VAD-fmk, $5 \mathrm{mM}$ EGTA, and/or $20 \mu \mathrm{M}$ BAPTA-AM, for $30 \mathrm{~min}$ followed by nsPEFs treatment. Cells were then quantified by immunoblot analysis using Odyssey for levels of Bid and t-Bid as described above. Caspase-dependence was determined by Bid cleavage that was inhibited by z-VAD-fmk. Calcium dependence was determined by Bid cleavage that was inhibited by EGTA and BAPTA-AM in the absence of z-VAD-fmk. Dependence on extracellular calcium was determined by Bid cleavage that was inhibited by EGTA in the presence of z-VAD-fmk. Dependence on total calcium was determined by Bid cleavage in the presence of EGTA and BAPTA-AM in the presence of z-VAD-fmk. Dependence on intracellular calcium was determined by the difference between total calcium and extracellular calcium responses.

Assessment of calpain activity in response to nsPEFs by fluorometric assay

Analysis of calpain catalytic activity was determined in total cell lysates (BioVision). Cell lysates were prepared and incubated with substrate (AC-LLY-AFC) and reaction buffer for $1 \mathrm{~h}$ at $37^{\circ} \mathrm{C}$ in the dark. Cleavage substrate fluorescence was measured by a microplate spectrofluorometer $(\mathrm{ex} / \mathrm{em}=488 / 520 \mathrm{~nm})$ with SoftMax Pro 5 software (Molecular Devices). A calpain-specific inhibitor (z-LLY-fmk) was used to identify calpain-specific substrate hydrolysis. Recombinant calpain I was used as positive control (data not shown).
Determination of intracellular reactive oxygen species (ROS) accumulations

E4 Cells were preloaded with $1 \mu \mathrm{M}$ CM- $\mathrm{H}_{2}$ DCFDA (5-6-chloromethy-2' $7^{\prime}$-dichlorodihydrofluorescein diacetate acetyl ester, Molecular Probes) in phenol red free DMEM media (Invitrogen) for $60 \mathrm{~min}$, and then pulsed with nsPEF as described previously [2]. Cells were analyzed by flow cytometry at $0,5,15,30$, and $60 \mathrm{~min}$ after pulsing. Cells incubated at $4^{\circ} \mathrm{C}$ for $1 \mathrm{~h}$ were used as positive controls. After removing from the cold, these cells were also sampled at $0,5,15,30$ and $60 \mathrm{~min}$.

Statistical analysis

Experiments were performed at least three times independently in this study. All the data is expressed as mean \pm standard error (SE). Statistical differences between control and treated groups were analyzed by student's $t$ test (twotailed) with $P<0.05$ regarded as statistically significant.

\section{Results}

NsPEFs induce caspase-correlated cell death in E4 squamous carcinoma cells (SCC) in vitro in an electric field amplitude-dependent manner

To determine cell viability after nsPEF treatment and whether nsPEFs activate caspase-associated cell death pathways in E4 SCC, cells were exposed to ten $300 \mathrm{~ns}$ pulses of $0-60 \mathrm{kV} / \mathrm{cm}$ electric field strengths. We chose ten, 300 ns pulses as parameters for this study so we could observe a complete range of electric field amplitudedependent effects on cell survival from little of no cell death to maximal cell death. Thus, we could observe various cell responses as possible mechanisms that were coincident with cell death. Cell viability was determined by counting trypan blue negative cells $24 \mathrm{~h}$ post pulse (Fig. 1 top panel). Caspase-3/7, $-8,-9$ activations were determined $2 \mathrm{~h}$ after nsPEF administration by flow cytometry using a cell permeable, irreversible inhibitors with selectivity towards specific caspase isoforms (Fig. 1 bottom panel). Results indicate an electric field-dependent decrease in cell viability that was correlated with electric field amplitudedependent increases in percentages of cells that exhibited active caspases. Analysis of caspase activation time courses indicated that they were activated between 20 and 60 min post pulse using the cell permeable, pan caspase irreversible inhibitor, z-VAD-fmk (data not shown). Therefore, nsPEF-induced E4 cell death is correlated with early caspase activation. Very few if any cells immediately 

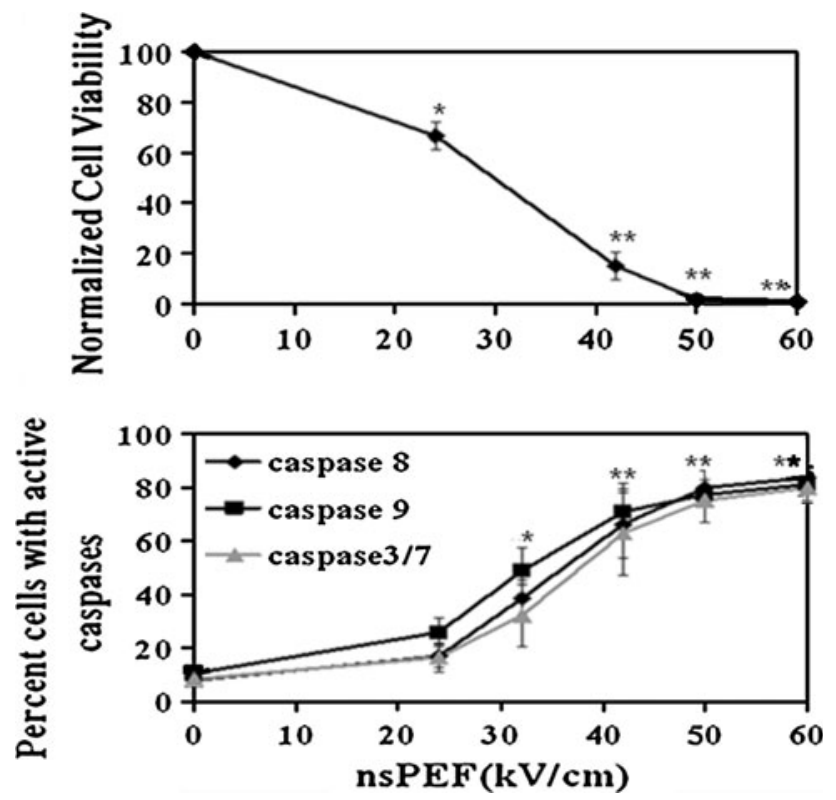

Fig. 1 NsPEF-induced cell death is correlated with the activation of caspase-3/7, -8 , and -9 in an electric field amplitude-dependent manner. E4 cells were pulsed ten times at $300 \mathrm{~ns}$ and electric field strengths between 0 and $60 \mathrm{kV} / \mathrm{cm}$. (Top panel) Cell viability was determined by counting trypan blue negative cells $24 \mathrm{~h}$ post pulse and normalizing values to the control. (Bottom panel) The percentage of cells with active caspase-3/7, -8, -9 was analyzed by flow cytometry using the cell permeable irreversible caspase inhibitors FAM-DEVD-fmk (caspase-3), FAM-LETD-fmk (caspase-8), FAM-LEHD-fmk (caspase-9) as described in Experimental Procedures. The values represent the mean $\pm \mathrm{SE}$ of three independent determinations. $* P<0.05$; ** $P<0.01$

lose plasma membrane integrity indicating that frank necrosis is not a mechanism of cell death.

Different sensitivities and heterogeneous responses in E4 cells to nsPEFs: electric field amplitudedependent effects on plasma membrane indicators, $\Delta \psi \mathrm{m}$, caspase activation and cytochrome c release

Figure 2 shows electric field thresholds for five different cell functional indicators in response to nsPEFs as defined by significant increases in cell numbers above basal values in the absence of electric field exposure. These cell responses also exhibited different temporal thresholds that were determined by time courses (data not shown) prior to experiments shown in Fig. 2. These values are indicated with each response. Significant numbers of cells showed increases in PI uptake and phosphatidylserine externalization at $24 \mathrm{kV} / \mathrm{cm}$, indicating changes in permeability and phospholipid orientation in plasma membranes. These plasma membrane responses were present as quickly as they could be measured (minutes) and were stable thereafter for at least $2 \mathrm{~h}$. Thus cells remained permeable, did not re-seal or reorient phosphatidylserine for at least $2 \mathrm{~h}$ after treatment. Figure 2 illustrates these analyses $15 \mathrm{~min}$ after pulsing. These effects on plasma membranes were electric field amplitude-dependent. Essentially all of the cells $(95-98 \%)$ were positive for both markers at electric fields $\geq 42 \mathrm{kV} / \mathrm{cm}$. Cell responses below $42 \mathrm{kV} / \mathrm{cm}$ were heterogeneous for both of these markers with only about 35 and $70 \%$ of cells showing positive responses at 24 and $32 \mathrm{kV} / \mathrm{cm}$, respectively. For effects on the mitochondria membrane potential, a significant increase in the numbers of cells that exhibited decreases in $\Delta \psi \mathrm{m}$ were observed at $24 \mathrm{kV} / \mathrm{cm}$, indicating depolarization of the inner mitochondria membrane. Increases in the numbers of cells that showed decreases in $\Delta \psi \mathrm{m}$ were seen within $10 \mathrm{~min}$, the earliest time measured. Figure 2 shows the response with TMRE 15 min post pulse. Similar results were observed with JC-1 (data not shown). Significant increases in the number of cells expressing active caspases occurred at $32 \mathrm{kV} / \mathrm{cm}$ and increased in an electric field amplitudedependent manner. Cell numbers with active caspases are shown in Fig. $21 \mathrm{~h}$ after pulsing. The temporal threshold for increases in cells positive for active caspases was between 20 and $60 \mathrm{~min}$. Cytochrome $c$ release required higher electric fields with significant increases in the numbers of cells releasing cytochrome $c$ occurring at $42 \mathrm{kV} / \mathrm{cm}$. Thus, cytochrome $c$ was the least sensitive

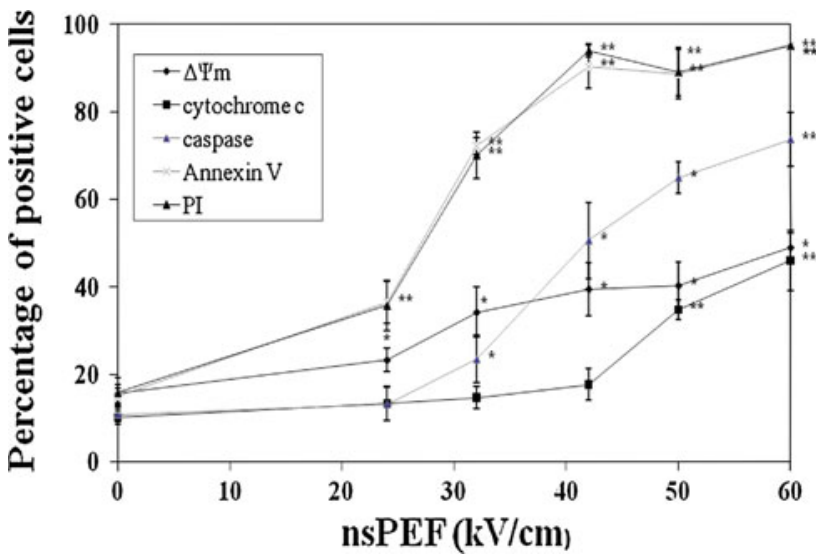

Fig. 2 Sensitivity thresholds for nsPEF effects on cell membranes, caspase activation and cytochrome $c$ release. E4 cells were exposed to 10 pulses at $300 \mathrm{~ns}$ ranging from 0 to $60 \mathrm{kV} / \mathrm{cm}$ and percentage of cells that showed significant responses were analyzed by flow cytometry for decreases in the $\Delta \psi \mathrm{m}$ with TMRE 15 min post pulse, active caspases with FITC-VAD-fmk $1 \mathrm{~h}$ post pulse, and cytochrome $c$ using the InnoCyte (Method I) $1 \mathrm{~h}$ post pulse. FITC-Annexin-V and PI were added immediately after nsPEF exposure and incubated for $15 \mathrm{~min}$ before analysis by flow cytometry. After initial measurements all responses were stable for a given electric field for at least $90 \mathrm{~min}$. Values are presented as percent cells with positive responses. The graph represents the mean $\pm \mathrm{SE}$ of three independent experiments. $* P<0.05$ and $* * P<0.01$ 
indicator tested, was not readily discernable before $1 \mathrm{~h}$ and only showed positive responses in about $45 \%$ of cells. The $1 \mathrm{~h}$ time point is shown in Fig. 2. We used two different assays for cytochrome $c$ release (see Experimental Procedures), both showing thresholds at $42 \mathrm{kV} / \mathrm{cm}$ and no significant differences between them at any electric field tested (data not shown). Notice that cell membrane responses (PI, Annexin-V and $\Delta \psi \mathrm{m}$ ) were evident at the earliest time tested while biological responses (caspase activation, cytochrome $c$ release) required more time. Also notice that at electric fields lower than $42 \mathrm{kV} / \mathrm{cm}$, cell numbers with increases in active caspase and decreases in $\Delta \psi \mathrm{m}$ were observed without significant cytochrome $c$ release. At electric fields greater than $50 \mathrm{kV} / \mathrm{cm}$, greater numbers of cells exhibited caspase activation than those with decreased $\Delta \psi \mathrm{m}$ and released cytochrome $c$. At electric fields $50 \mathrm{kV} / \mathrm{cm}$ and higher, equal numbers of cells exhibited decreases in $\Delta \psi \mathrm{m}$ and increases in cytochrome $c$ release and 30-40\% of cells did not show active caspases and about $55 \%$ did not show decreases in $\Delta \psi \mathrm{m}$ or increases in cytochrome $c$ release. Although the temporal thresholds were different for some of these responses, they were stable for at least 90 min under the conditions tested. Thus Fig. 2 can represent a $1 \mathrm{~h}$ snapshoot after treatment. However, cell responses indicate that these membrane and intracellular markers exhibit heterogeneous responses, different electric field sensitivities and different time courses.
Caspase-dependent and caspase-independent cytochrome $c$ release in response to nsPEFs

To determine whether cytochrome $c$ is released through the intrinsic pathway or type II cell extrinsic pathway, E4 cells were pretreated with $100 \mu \mathrm{M} \mathrm{z}$-VAD-fmk, a general caspase inhibitor, and then pulsed ten times at $300 \mathrm{~ns}, 60 \mathrm{kV} /$ $\mathrm{cm}$ in the presence of the inhibitor (Fig. 3). Inclusion of $1 \mathrm{mM}$ z-VAD-fmk gave identical results (data not shown), indicating that caspase activity was completely inhibited at $100 \mu \mathrm{M} \mathrm{z}$-VAD-fmk. Cytochrome $c$ release was determined $1 \mathrm{~h}$ post pulse by flow cytometry using Method I (see Experimental Procedures). Figure 3a shows a contour plot of a typical experiment with forward scatter on the Y-axis (FSC-A) and cytochrome $c$ fluorescence on the $\mathrm{X}$-axis (FITC-A). Cells that exhibit cytochrome $c$ release appear in the bottom right quadrant. Figure $3 b$ shows the combined results of three separate experiments with statistical comparisons. In sham treated control cells, very few cells show cytochrome $c$ release $(7.7 \pm 1.5 \%)$ and the caspase inhibitor has no significant effect $(9.5 \pm 2.2 \%)$. When cells were treated with ten $300 \mathrm{~ns}$ pulses at $60 \mathrm{kV} /$ $\mathrm{cm}, 46.2 \pm 2.5 \%$ of cells exhibited cytochrome $c$ release and the caspase inhibitor decreased that to $26.2 \pm 4.6 \%$. Three separate determinations gave similar results (Fig. 3b). Thus, based on the mean values in these experiments $43.3 \%$ of cytochrome $c$ release was caspase-dependent and $56.7 \%$

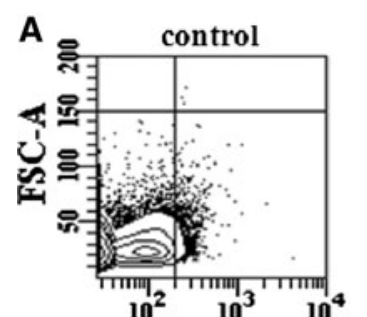

FITC-A

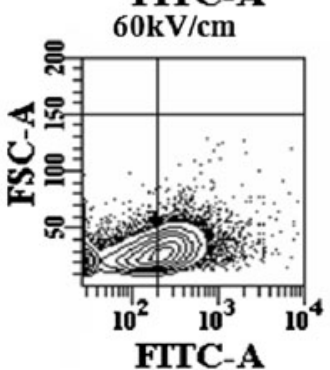

FITC-A

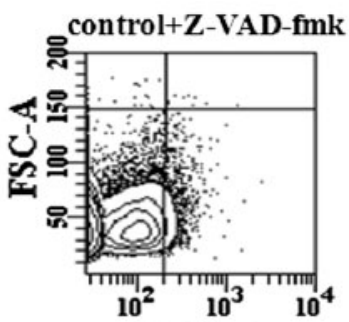

FTTC-A

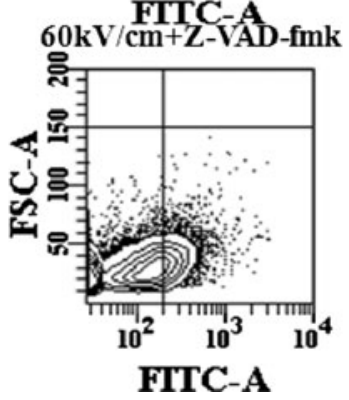

Fig. 3 A caspase inhibitor (z-VAD-fmk) partially decreases nsPEFinduced cytochrome $c$ release. E4 cells were pre-incubated with $100 \mu \mathrm{M} \mathrm{z}$-VAD-fmk, a pan caspase inhibitor, for $30 \mathrm{~min}$ and then pulsed ten times with $300 \mathrm{~ns}$ pulses at $60 \mathrm{kV} / \mathrm{cm}$ or sham treated. Cells were analyzed by flow cytometry for cytochrome $c$ release $1 \mathrm{~h}$ post pulse using Method I as described in Experimental Procedures. a Representative contour plots are shown with forward light scatter (FSC-A) on the $y$-axis and cytochrome $c$ fluorescence (FITC-A) on

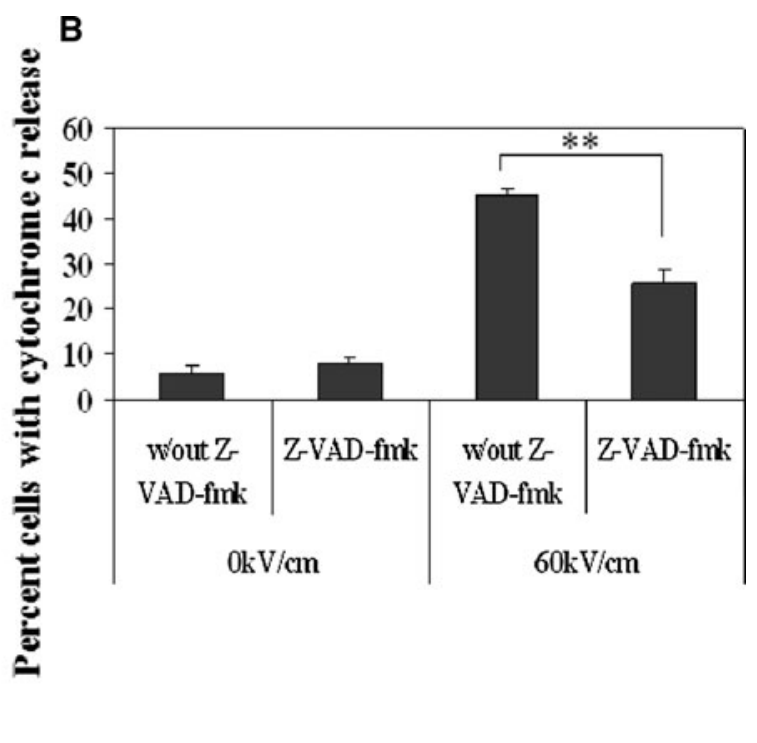

the $x$-axis. The top panels show sham treated control cells in the absence (left) and presence (right) of z-VAD-fmk. The bottom panels show cells treated with nsPEFs in the absence (left) and presence (right) of z-VAD-fmk. A decrease in cytochrome $c$ release is shown as a decrease in fluorescent intensity (shift from lower right quadrant to lower left quadrant) after preincubation with $100 \mu \mathrm{M}$ z-VAD-fmk. b The percentage of cells exhibiting cytochrome $c$ release $(n=3)$ is presented as mean $\pm \mathrm{SE}$. $* * P<0.01$ 
was caspase-independent, indicating more than one mechanism for cytochrome $c$ release in response to nsPEFs.

\section{NsPEFs generate Bid cleavage to t-Bid}

A well-characterized mechanism for caspase-dependent cytochrome $c$ release is through the cleavage of the BH3 only protein, Bid to the pro-apoptotic form t-Bid [24]. Such a possibility was also likely given that caspase activation was observed at lower electric fields and earlier times than cytochrome $c$ release. To determine if t-Bid was present, we analyzed cells that were exposed to ten $300 \mathrm{~ns}$ pulses at $60 \mathrm{kV} / \mathrm{cm}$ and then used immunoblot analysis to determine the quantities of Bid and t-Bid using quantitative Odyssey imaging at times after treatment with an antibody that recognized both Bid forms (Fig. 4). The left panel shows a typical immunoblot experiment of Bid and t-Bid in response to ten $300 \mathrm{~ns}$ pulses at $60 \mathrm{kV} / \mathrm{cm}$, a condition that resulted in significant cytochrome $c$ release (see Figs. 2, 3). The panels on the right show quantitative relationships of relative percentages of $\mathrm{t}-\mathrm{Bid}$ (top) and $\mathrm{Bid}$ (bottom) over a 60 min time course. In a time-dependent manner, there were increases in t-Bid and coordinate decreases in Bid. The sums of fluorescence intensities of both Bid forms

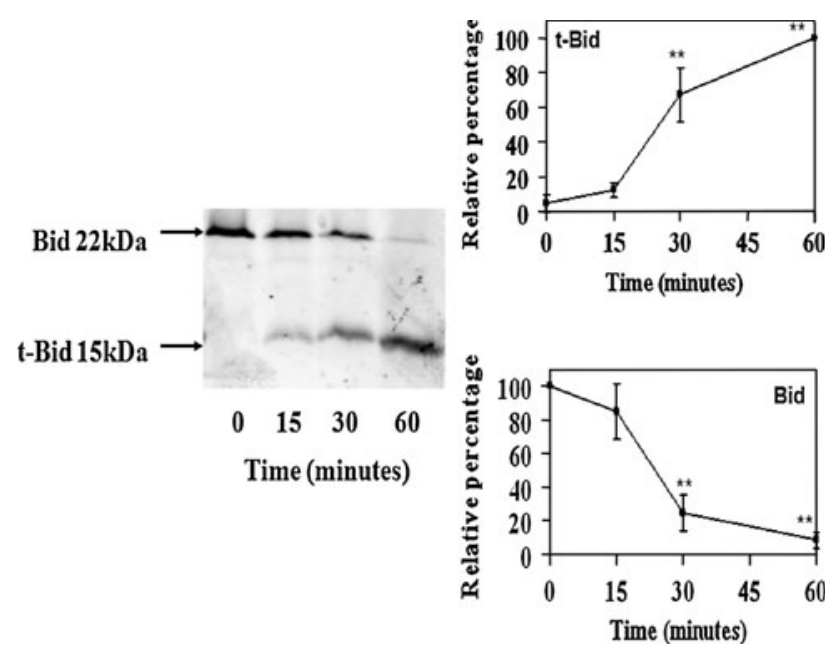

Fig. 4 NsPEFs induce Bid cleavage in a time-dependent manner in E4 cells. E4 cells were exposed with or without ten $300 \mathrm{~ns}$ pulses at $60 \mathrm{kV} / \mathrm{cm}$. Whole cell extracts $(75 \mu \mathrm{g}$ protein) were separated by SDS-PAGE, transferred to PVDF membranes and probed with an anti-Bid antibody. (Left panel) Bid (22 kDa) and t-Bid (15 kDa) levels were determined $0,15,30$ and $60 \mathrm{~min}$ post pulse by immunoblot analysis in a representative experiment as described in Experimental Procedures. Quantification was performed after normalizing Bid $(0 \mathrm{~min})$ or $\mathrm{t}$-Bid $(60 \mathrm{~min})$ to maximal levels. The right panel shows quantification of three independent experiments like the typical one shown in the left panel. The values in the top right and bottom right panels represent the relative percentages of t-Bid and Bid, respectively. Values $\geq 30 \mathrm{~min}$ are statistically significant $(* * P<0.01)$ were essentially the same at each time point, consistent with nsPEF-induced cleavage of Bid to generate proapoptotic t-Bid. At 60 min after treatment, nearly all Bid had been cleaved and pro-apoptotic t-Bid.

NsPEFs induce caspase-dependent and calciumdependent Bid cleavage; repetitive mechanisms for cytochrome $c$ release

The data in Fig. 3 using z-VAD-fmk indicated that nsPEFinduced cytochrome $c$ release, which is regulated at least in part by Bid cleavage, could be accounted for through caspase-dependent and caspase-independent mechanisms. Since nsPEFs induced-Bid cleavage and proteases other than caspases can cleave Bid, including calcium-dependent calpains [25, 26], we investigated the caspase- and calcium-dependency of Bid cleavage in response to nsPEFs (Fig. 5) as well as the possibility for nsPEF-induced calpain activity (Fig. 6). It was also of interest to determine whether the calcium was mobilized from intracellular stores, suggesting an intrinsic mechanism, or from extracellular media through plasma membranes as an extrinsic mechanism. Caspase-dependence was determined in the presence and absence of $\mathrm{z}$-VAD-fmk and the absence of EGTA and BAPTA. Determining caspase-dependent Bid cleavage in the presence of calcium was justified by the observations that caspase activity was shown to be independent of calcium [2, 3]. Calcium dependence was determined in the presence and/or absence of EGTA and BAPTA-AM. These analyses considered Bid cleavage to be either caspase- or calcium-dependent. To determine total calcium dependence from intracellular and extracellular compartments, caspases were inhibited by z-VADfmk. Thus, these values represent only the calcium component. The effect of extracellular calcium was determined in the presence and absence of EGTA and total calcium effects were determined in the presence and absence of EGTA and BAPTA. Intracellular calcium dependence was determined by the differences in total and extracellular calcium effects. The analyses were carried out in five to seven separate experiments by quantitative immunoblot analysis using Odyssey imaging of Bid experiments shown in Fig. 4. The results in Fig. 5 show that about $60 \%$ of Bid cleavage was attributed to caspases, while about $50 \%$ was attributed to calcium-dependent proteolysis. The differences are not statistically different, suggesting that effects of caspase- and calcium-dependent mechanisms contribute about equally to Bid cleavage. These slightly overlapping percentages could be due to a small amount of cross-dependence for caspase and calcium requirements, but are within reason of experimental variations. Further analyses indicated that $60-70 \%$ of the calcium contributing to Bid cleavage comes from extracellular 


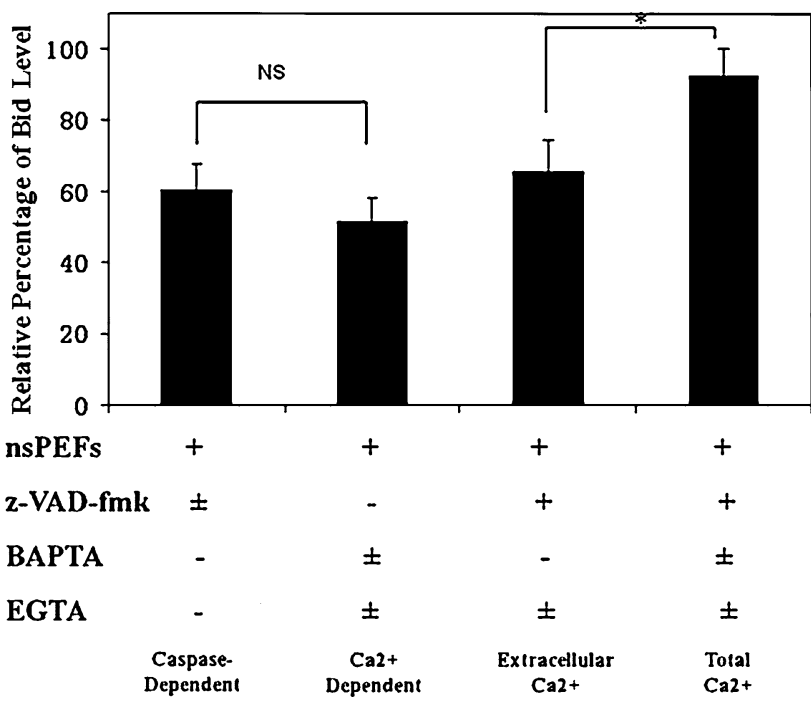

Fig. 5 Inhibition of Bid cleavage by calcium chelators EGTA and BAPTA and caspase inhibitor z-VAD-fmk: E4 cells were preincubated for $30 \mathrm{~min}$ in the presence or absence of EGTA $(5 \mathrm{mM}$, to chelate extracellular calcium), BAPTA-AM $(20 \mu \mathrm{M}$, to chelate intracellular calcium) and/or z-VAD-fmk (100 $\mu \mathrm{M}$ to inhibit caspase activation) prior to nsPEFs administration. Cells were exposed to ten $300 \mathrm{~ns}$ pulses at $60 \mathrm{kV} / \mathrm{cm}$ or sham treated and cell lysates were prepared $1 \mathrm{~h}$ post pulse as described in Experimental Procedures. Bid and t-Bid was quantified using Odyssey infrared imager (see Fig. 4) and normalized to total Bid levels in control. Caspase-dependent Bid cleavage is defined as the percentage of Bid cleavage that is inhibited by $\mathrm{z}$-VAD-fmk. Calcium-dependent Bid cleavage is defined as the percent of Bid cleavage that is inhibited by BAPTA and EGTA. To determine only calcium contributions, $z$-VAD-fmk was used to inhibit the caspase component. Total calcium-dependent (intracellular plus extracellular) Bid cleavage is defined as percentage of Bid cleavage that is inhibited by EGTA and BAPTA, but in the absence of caspasemediated Bid cleavage. Extracellular calcium-dependent Bid cleavage is defined as the percentage of Bid cleavage that is inhibited by EGTA, but in the absence of caspase-mediated Bid cleavage. Intracellular calcium-dependent Bid cleavage is defined as the difference between total calcium-dependent (intracellular plus extracellular) Bid cleavage and extracellular calcium-dependent Bid cleavage, all in the absence of caspase activity toward Bid. Values are expressed as mean $\pm \mathrm{SE}$ from five to seven experiments. $* P<0.05$

sources through plasma membranes and $30-40 \%$ comes from intracellular stores.

NsPEFs induce calpain activity

It was possibility that calcium-dependent Bid cleavage in response to nsPEFs was due to calpain activation. To test this, E4 cells were treated with ten $300 \mathrm{~ns}$ pulses at $60 \mathrm{kV} /$ $\mathrm{cm}$ in the presence or absences of BAPTA and EGTA, which inhibits intracellular and extracellular calciummediated events, respectively, and z-LLY-fmk, which specifically inhibits calpain activity. One hour after treatment cell extracts were prepared and assayed for calpain activity using the substrate AC-LLY-AFC. In the absence

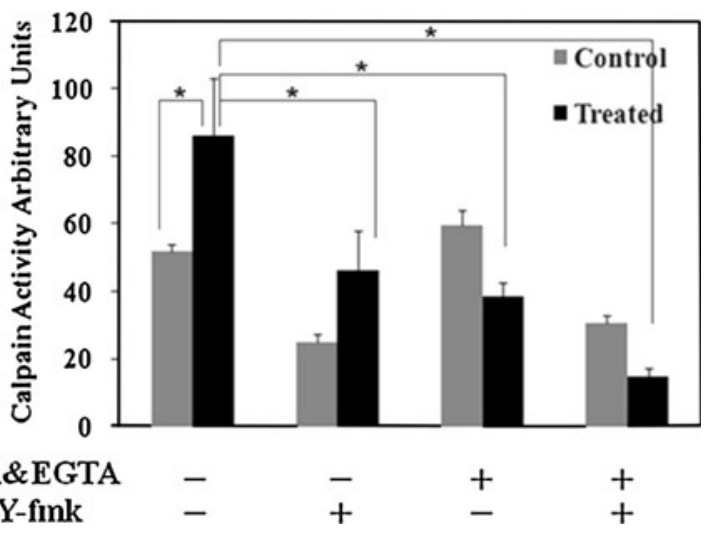

Fig. 6 nsPEFs induce calcium-dependent calpain activity in E4 cells. Calpain activity was determined by a calpain activity assay. E4 cells were pretreated with or without BAPTA-AM $(20 \mu \mathrm{M})$ and EGTA $(5 \mathrm{mM})$ for $30 \mathrm{~min}$ followed by exposing to ten $300 \mathrm{~ns}$ at $60 \mathrm{kV} / \mathrm{cm}$ or left untreated. Cells were then lysed and calpain activity was determined with or without adding calpain inhibitor z-LLY-fmk $(200 \mu \mathrm{M})$ to lysates. Data are expressed as mean $\pm \mathrm{SE}$ of three independent experiments. ${ }^{*} P<0.05$

of these inhibitors/chelators, basal calpain activity was about 50 units and nsPEFs increased this activity 1.7-fold. In the presence of the calpain inhibitor, both basal and nsPEF-stimulated activities were decreased by about $50 \%$. In the presence of BAPTA and EGTA, basal activity was not significantly affected, but nsPEF-stimulated activity was inhibited by $>50 \%$. When calcium chelators and the calpain inhibitor were present, basal activity was not inhibited any greater than when the calpain inhibitor was present alone. NsPEF-stimulated activity was inhibited by $>80 \%$. This data indicate that E4 cells exhibit calciuminsensitive basal calpain activity and calcium-sensitive nsPEF-stimulated activity. Although not tested here, these data suggest that other protease activities might be activated by nsPEFs.

NsPEFs induce cell death without ROS generation or changes in pro-survival protein $\mathrm{Bcl} 2$ and $\mathrm{Bcl}-\mathrm{xl}$

To determine whether ROS generation contributes to nsPEF-induced apoptosis, E4 cells were pulsed in the presence of $\mathrm{CM}-\mathrm{H}_{2}$ DCFDA and mean fluorescence intensity was analyzed by flow cytometry $0,5,15,30,60 \mathrm{~min}$ post pulse (Fig. 7). Treatment of cells for $1 \mathrm{~h}$ at $4{ }^{\circ} \mathrm{C}$ increased ROS by fivefold. ROS levels quickly decreased in a time-dependent manner to near control levels by $1 \mathrm{~h}$, demonstrating significant antioxidant activity in E4 SCC. In response to nsPEFs E4 cells show a slight electric field amplitude-dependent increases that were not statistically significant. Unlike the positive control, no time-dependent decreases in ROS levels were observed after pulsing. These results suggest that nsPEFs have minimal effects ROS 


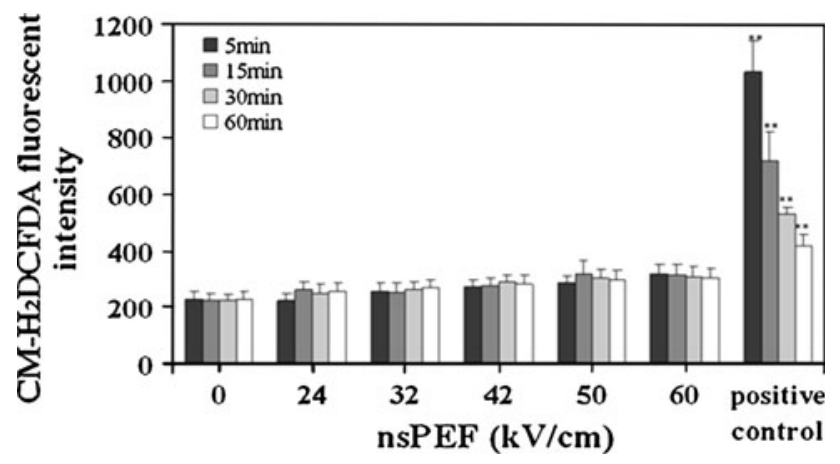

Fig. 7 Measurements of ROS generation in E4 cells after nsPEFs exposure. E4 cells were preincubated with ROS indicator CM$\mathrm{H}_{2}$ DCFDA for $60 \mathrm{~min}$ and exposed to ten pulses at $300 \mathrm{~ns}$ between 0 and $60 \mathrm{kV} / \mathrm{cm}$. Intracellular ROS formation was evaluated by measuring fluorescent intensity $5,15,30,60 \mathrm{~min}$ post pulse by flow cytometer. For the positive controls cells incubated at $4 \mathrm{C}$ for $1 \mathrm{~h}$ and removed at the indicated time for measurement of ROS levels. The graph represents the mean $\pm \mathrm{SE}$ of three independent experiments. ** $P<0.01$ for differences at the indicated time compare to the control at $0 \mathrm{kV} / \mathrm{cm}$

generation and that E4 cells exhibit active antioxidant activities, which is often typical for cancer cells.

\section{NsPEF have no significant effects of Bcl-2 or Bcl-xl} levels

In a further analysis of nsPEF effects, levels of the antiapoptotic proteins $\mathrm{Bcl}-2$ and $\mathrm{Bcl}-\mathrm{xl}$ were analyzed in the absence and presence of nsPEF treatment in E4 cells using quantitative immunoblot analysis with Odyssey imaging (data not shown). Both $\mathrm{Bcl} 2$ and $\mathrm{Bcl}-\mathrm{xl}$ were present a single bands in control cells. There were no effects of ten $300 \mathrm{~ns}$ pulses at $60 \mathrm{kV} / \mathrm{cm}$ on either anti-apoptotic protein with the first hour after treatment with nsPEFs, suggesting that anti-apoptotic protein levels were unaffected while a pro-apoptotic protein, Bid was activated.

\section{Discussion}

Evidence from E4 SCC gives new insight and understanding for cell death and apoptosis-like mechanisms and their origin in response to nsPEFs. In the experiments presented here we quantified a diverse set of cell responses to nsPEFs in E4 SCC. The results indicate that cell death can be dependent and/or independent of caspase activity, calpain activity, calcium from intracellular stores or extracellular media, cytochrome $c$ release and decreases in $\Delta \psi \mathrm{m}$. Thus, nsPEFs initiated charge-mediated events in plasma membranes and intracellular membranes through multiple signaling mechanisms inducing signatures of apoptosis and other forms of cell death. Even at the highest electric fields investigated, frank necrosis as defined by immediate plasma membrane rupture was not present, so cell death is driven by biological responses that appear to be, at least in part, programmed. Ibey et al. [27] found that apoptosis (DNA fragmentation of agarose gels) was present in U937 cells exposed to 600 pulses at $10 \mathrm{~ns}$ and $100 \mathrm{kV} /$ $\mathrm{cm}(700 \mathrm{~J} / \mathrm{g})$; however, necrosis was a major form of cell death. However, in this study the cell type was different and the energy absorbed or dose $(\mathrm{J} / \mathrm{g})$ for the pulses was about seven times higher than in the present study $(\sim 700$ vs. $\sim 100 \mathrm{~J} / \mathrm{g}$ ). It should be pointed out that factors other than energy absorbed are likely involved to determine nsPEF effects [28].

After treatment with nsPEFs caspases are activated at earlier times and at lower electric fields than cytochrome $c$ release, demonstrating initial activation of a type I cell extrinsic pathway. This is similar to results in nsPEF treated HCT-116 colon carcinoma cells [3], but different than B16f10 cells that did not release cytochrome $c$ [2] and Jurkat cells with concomitant cytochrome $c$ release and caspase activation [1]. This clearly defines cell type-specific response to nsPEF-induced cell death. Unlike the other analyzes, here we show for the first time caspasedependent and caspase-independent cytochrome $c$ release, demonstrating the additional presence of a type II cell extrinsic pathway through Bid cleavage for cytochrome $c$ release. Caspase-dependent (55-60\%) and calciumdependent (40-45\%) Bid cleavage pathways were demonstrated with quantitative and reciprocal increases in t-Bid and decreases in Bid. Further, for calcium-dependent Bid cleavage, extracellular calcium $(\sim 70 \%)$ played a predominant role compared to calcium from intracellular stores $(\sim 30 \%)$. Caspase-independent Bid cleavage is most likely accomplished by charge-mediated interventions with calcium signaling from and/or nanoporation in the endoplasmic reticulum, capacitative calcium entry $[5,22]$ and/ or calcium entry through nanopores in plasma membranes [19]. Since caspase activation has been shown to be calcium-independent $[2,3]$, calcium-dependent Bid cleavage appears to be due to calpain activation. This was confirmed by showing calcium-sensitive calpain activity was specifically inhibited by the calpain inhibitor z-LLY-fmk. Taken together, these results show for the first time nsPEF-mediated at least two mechanisms for Bid cleavage and cytochrome $c$ release-one caspase-dependent and one calpain-dependent. While there are reports of cross-talk and/or synergistic behaviors between calpains and caspases $[29,30]$, since the sum of caspase-dependent and calpaindependent Bid cleavage activities, which were determined independently of the other, equal about $100 \%$ of total Bid cleavage. Generation of $\mathrm{t}$-Bid, a critical point connecting extrinsic and intrinsic pathways, by at least two different protease families provides beneficial repetitive 
mechanisms to maximize cytochrome $c$ release and apoptosis induction through mitochondria pathways. Thus, nsPEFs promote pro-apoptotic signals that overcome resistances to apoptosis, which are present in most if not all cancers, thereby potentially abolishing the universal cancer hallmark of apoptosis evasion [31].

By analyzing several different $\mathrm{E} 4$ cell responses to nsPEFs by flow cytometry, it was possible to establish several other characteristics for nsPEF-induced cell death. Under nsPEF conditions that eliminated $\geq 95 \%$ of E4 SCC, only $50 \%$ of cells exhibited cytochrome $c$ release and decreases in $\Delta \psi \mathrm{m}$, while $75-80 \%$ of cells exhibited active caspases. Moreover, each of these responses showed different thresholds or sensitivities among themselves, with cytochrome $c$ release as the least sensitive response. Sensitivities for plasma and inner mitochondria membrane permeability appeared to be similar, although plasma membrane reactions as determined by PI uptake and PS externalization were overall more responsive than inner mitochondria membrane as determined by decreases in $\Delta \psi \mathrm{m}$. These are most likely due to nanopore formation in the respective membranes. These results are consistent with an overall greater initial response from plasma membrane sensitivity leading to activation of extrinsic apoptosis mechanisms compared to intrinsic mechanisms from mitochondria membrane sensitivity. An extrinsic mechanism is also consistent with a greater response from extracellular calcium and a lesser response from intracellular calcium.

The predominance of extrinsic mechanisms with greater effects on plasma membranes and from extracellular calcium-dependent responses compared to intrinsic mechanisms measured by decreases in $\Delta \psi \mathrm{m}$ and intracellular calcium-dependent responses can possibly be explained by characteristics of electric fields pulses. Pulsed electric field requirements for intracellular membrane effects include a fast pulse rise time, high electric fields, and a pulse duration that is shorter than the relaxation time of intracellular membrane, which is on the order of $\sim 100 \mathrm{~ns}$ [16]. While pulses used here have sufficient rise times and electric fields ( $\leq 4 \mathrm{~ns}$ and $60 \mathrm{kV} / \mathrm{cm}$, respectively), the relatively long $300 \mathrm{~ns}$ pulse duration may not be short enough to allow fully effective intracellular effects. While the mechanism(s) for caspase activation remains to be defined, nsPEF effects are due to membrane charging events. While PI uptake occurs in $>95 \%$ of cells, and cytochrome $c$ is released in about $50 \%$ of cells, caspases are activated in $75-80 \%$ of cells. This suggests that charging of the plasma membrane may be more closely tied to caspase activation than charging of intracellular membranes. This is consistent with caspase activation occurring before cytochrome $c$ release, reminiscent of the type I cell extrinsic mechanism, and greater numbers of cells exhibiting PI uptake at lower electric fields than cells with decreased $\Delta \psi \mathrm{m}$. At the highest electric field tested $(60 \mathrm{kV} / \mathrm{cm})$, all of these $n s P E F-$ induced responses are coincident with cell death in $>95 \%$ of cells indicating heterogeneous cell responses in nsPEFinduced cell death. Given that $\sim 75-80 \%$ of cells express active caspases, caspase-associated cell death appears to be a predominant event. Thus, cell death occurs against different backgrounds of mitochondria responses in $\Delta \psi \mathrm{m}$, cytochrome $c$ release and caspase activation. While decreases in $\Delta \psi \mathrm{m}$, caspase activation and cytochrome $c$ release may all occur within given populations of cells, other cell populations may exhibit one or more of these, most notably through the cytochrome $c$-independent extrinsic pathway(s). Thus, nsPEF-induced apoptosis mechanisms are heterogeneous within E4 SCC populations as well as heterogeneous among cell types [1, 3].

Special consideration should be made for using PI uptake as a cell viability marker and phosphatidylserine externalization as an apoptosis marker in response to electric field stimulation. Using PI as a viability marker could be misleading given that electric fields have transient and reversible effects on membrane permeability. Some cells can survive after conventional electroporation as well as after sub-lethal nsPEF conditions [32]. Thus, to avoid possible erroneous conclusions when evaluating effects of electric field on plasma membranes it is not advisable to use PI uptake as a viability marker. In the studies here, $300 \mathrm{~ns}$ pulse durations are long enough to allow plasma membrane pores to expand so they are large enough to include PI, which is on the order of about a nanometer. Nevertheless, the pulse durations are short and fast enough to induce intracellular membrane effects. Moreover, modeling evidence indicates that nsPEFs can "pull" phosphatidylserine through nanopores [33-37] acting as an apoptotic mimic that in the presence of macrophages was shown to cause phagocytosis of caspase-negative cells [38]. Thus, to avoid possible erroneous conclusions, phosphatidylserine externalization should not be used as an apoptosis marker or used very cautiously when evaluating effects of electric fields on plasma membranes. Particularly, PI and Annexin-V should not be used alone as indicators for viability/necrosis and apoptosis, respectively, when evaluating effects of electric fields or other stimuli. In addition, defining apoptosis characteristic in vitro can be complicated by the presence of artifacts that are not present during apoptosis in vivo. In this study, events within the first $2 \mathrm{~h}$ were a primary focus to avoid or lessen these issues. While time courses for cell death are stimulidependent, nsPEF effects occur relatively rapidly in E4 cells. What can be discerned in this time fame, especially within the first hour(s), is reported in this study.

There may be a case made for a unique type of nsPEFinduced cell death. Cell death initiated by nsPEFs can 
be differentiated from TNF-induced necroptosis, $\mathrm{H}_{2} \mathrm{O}_{2}$ induced necrosis and anti-Fas-induced secondary necrosis [39] in that nsPEF, in contrast to cold exposure, did not exhibit ROS generation determined with $\mathrm{CM}-\mathrm{H}_{2}$ DCFDA and did not lead to hyperpolarization of $\Delta \psi \mathrm{m}$ as the other stimuli-induced cell death did. Thus, E4 SCC mechanisms that protect against DNA and other damage from ROS are not expected to be effective in response to nsPEFs. Given that electric fields with nsPEF characteristics do not exist in nature, nsPEFs provide a distinct cell death stimulus, which could account for the heterogeneity of nsPEF-induced responses in E4 SCC.

In $\mathrm{E} 4$ cells, new evidence is provided that nsPEFs act through multiple mechanisms to induce cell death at least in part due to apoptosis through extrinsic type I, extrinsic type II and/or intrinsic pathway(s). Based on several lines of evidence, the type I extrinsic pathway appears to be predominant among these. While calpain activation can be initiated by mobilization of calcium from intracellular and/ or extracellular sources by nanopore formation, it remains to be determined how a presumed plasma membrane stimulus leads to caspase activation. The extrinsic pathway is activated by plasma membrane death receptors. It is possible that electric field interactions with the plasma membrane could activate these receptors either through nanopore formation or some other charging event that rearranges lipid bilayers. Alternatively, changes in membrane permeability could alter the intracellular milieu, such as apoptosis volume decrease [40], leading to caspase activation. Aside from caspase activation other mechanisms are likely to contribute to nsPEF-induced cell death. Since necrosis induced by immediate plasma membrane rupture is not a significant cause of E4 SCC cell death, nsPEFs must set into motion events that are mediated by biological mechanisms in response to nsPEF-mediated stresses that are at least in part programmed.

Open Access This article is distributed under the terms of the Creative Commons Attribution Noncommercial License which permits any noncommercial use, distribution, and reproduction in any medium, provided the original author(s) and source are credited.

\section{References}

1. Beebe SJ, Fox PM, Rec LJ, Willis EL, Schoenbach KH (2003) Nanosecond, high-intensity pulsed electric fields induce apoptosis in human cells. FASEB J 17:1493-1495

2. Ford WE, Ren W, Blackmore PF, Schoenbach KH, Beebe SJ (2010) Nanosecond pulsed electric fields stimulate apoptosis without release of pro-apoptotic factors from mitochondria in B16f10 melanoma. Arch Biochem Biophys 497:82-89

3. Hall EH, Schoenbach KH, Beebe SJ (2007) Nanosecond pulsed electric fields induce apoptosis in p53-wildtype and p53-null HCT116 colon carcinoma cells. Apoptosis 12:1721-1731
4. Beebe S, Fox P, Rec L, Somers K, Stark R, Schoenbach K (2002) Nanosecond pulsed electric field (nsPEF) effects on cells and tissues: apoptosis induction and tumor growth inhibition. IEEE Trans Plasma Sci 30:286-292

5. Beebe SJ, Blackmore PF, White J, Joshi RP, Schoenbach KH (2004) Nanosecond pulsed electric fields modulate cell function through intracellular signal transduction mechanisms. Physiol Meas 25:1077-1093

6. Beebe SJ, White J, Blackmore PF, Deng Y, Somers K, Schoenbach KH (2003) Diverse effects of nanosecond pulsed electric fields on cells and tissues. DNA Cell Biol 22:785-796

7. Chen X, Kolb JF, Swanson RJ, Schoenbach KH, Beebe SJ (2010) Apoptosis initiation and angiogenesis inhibition: melanoma targets for nanosecond pulsed electric fields. Pigment Cell Melanoma Res 23:554-556

8. Kerr JF, Wyllie AH, Currie AR (1972) Apoptosis: a basic biological phenomenon with wide-ranging implications in tissue kinetics. Br J Cancer 26:239-257

9. Danial NN, Korsmeyer SJ (2004) Cell death: critical control points. Cell 116:205-219

10. Kumar S (2007) Caspase function in programmed cell death. Cell Death Differ 14:32-43

11. Lamkanfi M, Festjens N, Declercq W, Vanden Berghe T, Vandenabeele P (2007) Caspases in cell survival, proliferation and differentiation. Cell Death Differ 14:44-55

12. Ferri KF, Kroemer G (2001) Organelle-specific initiation of cell death pathways. Nat Cell Biol 3:E255-E263

13. Kroemer G, Galluzzi L, Vandenabeele P et al (2009) Classification of cell death: recommendations of the Nomenclature Committee on Cell Death. Cell Death Differ 16:3-11

14. Gowrishankar TR, Esser AT, Vasilkoski z, Smith KC, Weaver JC (2006) Microdosimetry for conventional and supra-electroporation in cells with organelles. Biochem Biophys Res Commun 341: 1266-1276

15. Stewart D Jr, Gowrishankar T, Weaver J (2004) Transport lattice approach to describing cell electroporation: use of a local asymptotic model. IEEE Trans Plasma Sci 32:1696-1708

16. Schoenbach KH, Beebe SJ, Buescher ES (2001) Intracellular effect of ultrashort electrical pulses. Bioelectromagnetics 22:440-448

17. Joshi R, Hu Q, Schoenbach K (2004) Modeling studies of cell response to ultrashort, high-intensity electric fields-implications for intracellular manipulation. IEEE Trans Plasma Sci 32:1677

18. Kotnik T, Miklavcic D (2006) Theoretical evaluation of voltage inducement on internal membranes of biological cells exposed to electric fields. Biophys J 90:480-491

19. Pakhomov AG, Bowman AM, Ibey BL, Andre FM, Pakhomova ON, Schoenbach KH (2009) Lipid nanopores can form a stable, ion channel-like conduction pathway in cell membrane. Biochem Biophys Res Commun 385:181-186

20. Bowman AM, Nesin OM, Pakhomova ON, Pakhomov AG (2010) Analysis of plasma membrane integrity by fluorescent detection of $\mathrm{Tl}(+)$ uptake. J Membr Biol 236:15-26

21. Tekle E, Oubrahim H, Dzekunov SM, Kolb JF, Schoenbach KH, Chock PB (2005) Selective field effects on intracellular vacuoles and vesicle membranes with nanosecond electric pulses. Biophys J 89:274-284

22. White JA, Blackmore PF, Schoenbach KH, Beebe SJ (2004) Stimulation of capacitative calcium entry in HL-60 cells by nanosecond pulsed electric fields. J Biol Chem 279:22964-22972

23. Pakhomov AG, Phinney A, Ashmore J, Walker K III, Kolb JF, Kono S, Schoenbach KH, Murphy MR (2004) Characterization of the cytotoxic effect of high-intensity, 10-ns duration electrical pulses. IEEE Trans Plasma Sci 32:1579-1586

24. Luo X, Budihardjo I, Zou H, Slaughter C, Wang X (1998) Bid, a $\mathrm{Bcl} 2$ interacting protein, mediates cytochrome $c$ release from 
mitochondria in response to activation of cell surface death receptors. Cell 94:481-490

25. Chen M, He H, Zhan S, Krajewski S, Reed JC, Gottlieb RA (2001) Bid is cleaved by calpain to an active fragment in vitro and during myocardial ischemia/reperfusion. J Biol Chem 276:30724-30728

26. Mandic A, Viktorsson K, Strandberg L et al (2002) Calpainmediated Bid cleavage and calpain-independent Bak modulation: two separate pathways in cisplatin-induced apoptosis. Mol Cell Biol 22:3003-3013

27. Ibey BL, Pakhomov AG, Gregory BW, Khorokhorina VA, Roth CC, Rassokhin MA, Bernhard JA, Wilmink GJ, Pakhomova ON (2010) Selective cytotoxicity of intense nanosecond-duration electric pulses in mammalian cells. Biochim Biophys Acta 1800: 1210-1219

28. Schoenbach KH, Joshi RP, Beebe SJ, Baum CE (2009) A scaling law for membrane permeabilization with nanopulses. IEEE Trans Dielectr Electr Insul 16:1224-1235

29. Nakagawa T, Yuan J (2000) Cross-talk between two cysteine protease families. Activation of caspase-12 by calpain in apoptosis. J Cell Biol 150:887-894

30. Neumar RW, Xu YA, Gada H, Guttmann RP, Siman R (2003) Cross-talk between calpain and caspase proteolytic systems during neuronal apoptosis. J Biol Chem 278:14162-14167

31. Hanahan D, Weinberg RA (2000) The hallmarks of cancer. Cell 100:57-70

32. Hall EH, Schoenbach KH, Beebe SJ (2007) Nanosecond pulsed electric fields have differential effects on cells in the S-phase. DNA Cell Biol 26:160-171
33. Vernier PT, Sun Y, Marcu L, Craft CM, Gundersen MA (2004) Nanoelectropulse-induced phosphatidylserine translocation. Biophys J 86:4040-4048

34. Hu Q, Joshi RP, Schoenbach KH (2005) Simulations of nanopore formation and phosphatidylserine externalization in lipid membranes subjected to a high-intensity, ultrashort electric pulse. Phys Rev E Stat Nonlin Soft Matter Phys 72:031902

35. Vernier PT, Ziegler MJ, Sun Y, Gundersen MA, Tieleman DP (2006) Nanopore-facilitated, voltage-driven phosphatidylserine translocation in lipid bilayers-in cells and in silico. Phys Biol 3:233-247

36. Weaver JB, Rauwerdink AM, Sullivan CR, Baker I (2008) Frequency distribution of the nanoparticle magnetization in the presence of a static as well as a harmonic magnetic field. Med Phys 35:1988-1994

37. Smith KC, Weaver JC (2008) Active mechanisms are needed to describe cell responses to submicrosecond, megavolt-per-meter pulses: cell models for ultrashort pulses. Biophys J 95:1547-1563

38. Tekle E, Wolfe MD, Oubrahim H, Chock PB (2008) Phagocytic clearance of electric field induced 'apoptosis-mimetic' cells. Biochem Biophys Res Commun 376:256-260

39. Berghe TV, Vanlangenakker N, Parthoens E et al (2010) Necroptosis, necrosis and secondary necrosis converge on similar cellular disintegration features. Cell Death Differ 17:922-930

40. Bortner CD, Cidlowski JA (2002) Apoptotic volume decrease and the incredible shrinking cell. Cell Death Differ 9:1307-1310 